\title{
Phase transition and critical end point driven by an external magnetic field in asymmetric quark matter
}

\author{
Pedro Costa, ${ }^{1}$ Márcio Ferreira, ${ }^{1}$ Hubert Hansen, ${ }^{2}$ Débora P. Menezes, ${ }^{3}$ and Constança Providência ${ }^{1}$ \\ ${ }^{1}$ Centro de Física Computacional, Department of Physics, University of Coimbra, \\ P-3004-516 Coimbra, Portugal \\ ${ }^{2} I P N L$, Université de Lyon/Université Lyon 1, CNRS/IN2P3, 4 rue E.Fermi, \\ F-69622 Villeurbanne Cedex, France \\ ${ }^{3}$ Departamento de Física, CFM, Universidade Federal de Santa Catarina, \\ Florianópolis, SC, CP 476, CEP 88.040-900, Brazil
}

(Received 5 August 2013; published 25 March 2014)

\begin{abstract}
The location of the critical end point (CEP) in the QCD phase diagram is determined under different scenarios. The effect of strangeness, isospin/charge asymmetry and an external magnetic field is investigated. The discussion is performed within the $2+1$ flavor Nambu-Jona-Lasinio model with Polyakov loop. It is shown that isospin asymmetry shifts the CEP to larger baryonic chemical potentials and smaller temperatures. At large asymmetries the CEP disappears. However, a strong enough magnetic field drives the system into a first order phase transition.
\end{abstract}

DOI: 10.1103/PhysRevD.89.056013

PACS numbers: 24.10.Jv, 11.10.-z, 25.75.Nq

\section{INTRODUCTION}

Presently the study of the phase diagram of QCD is the subject of both theoretical and experimental studies under extreme conditions of density and temperature. In particular, it is expected that the phenomenon of deconfinement occurs in relativistic heavy-ion collisions and in the interior of compact stars, two very different scenarios when isospin asymmetry is considered. While in heavy ion collisions (HIC) the proton fraction is presently not smaller than $\sim 0.4$, and much smaller proton fractions are expected in the interior of neutron stars. The effect of isospin/charge asymmetry in the QCD phase diagram has recently been discussed in [1], and it was shown that for a sufficiently asymmetric system the critical end point (CEP) is not present [1,2].

Another degree of freedom that must be considered when discussing the QCD phase diagram is strangeness. In the interior of a neutron star it is expected that strangeness is present in the form of hyperons, of a kaon condensate or of a core of deconfined quark matter. $\beta$ equilibrium is energetically favored, and the Fermi pressure of neutrons is reduced if strangeness degrees of freedom are generated through the action of the weak interaction. On the other hand, the strong force governs heavy ion collisions.

The compact astrophysical objects known as magnetars [3], which include soft gamma repeaters and anomalous $\mathrm{x}$-ray pulsars, are expected to have very strong magnetic fields in their interior. Extremely strong magnetic fields are also expected to affect the measurements in heavy ion collisions at very high energies [4] or the behavior of the first phases of the Universe [5]. Fields of this intensity affect the QCD phase diagram as shown in [6]. Therefore, understanding the effect of an external magnetic field on the structure of the QCD phase diagram is very important, and this has already led to several studies [7-13], in particular, at zero chemical potential $\mu=0$ (the $T-e B$ plane); see [14-17] for a review.

At zero chemical potential, almost all low-energy effective models, including the Nambu-Jona-Lasinio (NJL)type models, as well as some lattice QCD (LQCD) calculations [18-22], found an enhancement of the condensate due to the magnetic field (magnetic catalysis) independently of the temperature. However, more recently, LQCD studies [16,23], for $N_{f}=2+1$ flavors with physical quarks and pion masses, show a suppression of the light condensates by the magnetic field in the transition temperature region, an effect known as inverse magnetic catalysis [24-26]. Indeed, near the transition temperatures the condensate shows a nonmonotonic behavior decreasing with $e B$. Also interesting is the fact that new lattice QCD calculations report a rise of the Polyakov loop with $e B$ at the pseudocritical temperature and $e B \lesssim 0.8 \mathrm{GeV}^{2}$ indicating an inverse magnetic catalysis [20]. However, as pointed out by the authors at a sufficiently strong magnetic field strength the magnetic catalysis is seen to be in agreement with almost all effective models that predict magnetic catalysis at any temperature and magnetic field strength like the Nambu-Jona-Lasinio model with Polyakov loop (PNJL).

In [27], it has been shown that within the entangled Nambu-Jona-Lasinio model with Polyakov loop (EPNJL) [28] the inverse magnetic catalysis at $\mu=0$ could be reproduced with a magnetic field dependent parameter $T_{0}(e B)$ in the Polyakov loop. The magnetic field dependence of this parameter mimics the reaction of the gluon sector to the presence of an external magnetic field.

The inverse magnetic catalysis mechanism does not occur only at $\mu=0$ and large temperatures. This 
phenomenon has also been obtained at finite chemical potential and zero or low temperatures: the critical chemical potential for the phase transition decreases with increasing $e B$. This is, however, a region of the QCD phase diagram still not accessible to LQCD. In the NJL model the first studies were performed in Ref. [29] at $T=0$ and in Ref. [30] for the full $T-\mu-B$ case. This effect has also been obtained in other models $[6,31]$ and is the result of a competition between the decrease of the free energy due to the condensation in the magnetic field and the increase of the free energy due to the accommodation of more valence quarks in the phase space [31]. In the present work, the same effect will be obtained. In this context, QCD-like models are very useful in the region of moderate temperature and chemical potential in the presence of an external magnetic field.

In the present work we investigate several scenarios of interest for the study of either heavy ion collisions or compact stars. We show how the CEP changes with the isospin asymmetry and confirm previous results obtained within other models that indicate that at sufficiently high asymmetry it does not exist $[1,2,32]$. We also consider the effect of strangeness in the QCD phase diagram by analyzing different chemical equilibrium conditions. Finally, we calculate the effect of an external magnetic field on the same scenarios previously discussed for a nonmagnetized system. It will be shown that the magnetic field, if sufficiently strong, can drive a first order phase transition in an isospin asymmetric system at a quite low temperature. The discussion is performed within the $2+1$ flavor PNJL [33], and for reference some results calculated within the NJL model are also included.

\section{MODEL AND FORMALISM}

Most of the properties of the quark condensates in the presence of an external magnetic field were previously obtained with the two flavor version of the PNJL and EPNJL models [15,34]. Recently, the effects of an external magnetic field on the fluctuations and correlations of the quark number and conserved charges were studied in the $2+1$ PNJL model [35].

In the present work we describe the quark matter subject to strong magnetic fields within the $2+1$ PNJL model. The PNJL Lagrangian with explicit chiral symmetry breaking where the quarks couple to a (spatially constant) temporal background gauge field, represented in terms of the Polyakov loop and in the presence of an external magnetic field, is given by [33]

$$
\begin{aligned}
\mathcal{L}= & \bar{q}\left[i \gamma_{\mu} D^{\mu}-\hat{m}_{f}\right] q+\mathcal{L}_{\mathrm{sym}}+\mathcal{L}_{\mathrm{det}} \\
& +\mathcal{U}(\Phi, \bar{\Phi} ; T)-\frac{1}{4} F_{\mu \nu} F^{\mu \nu},
\end{aligned}
$$

where the quark sector is described by the $\mathrm{SU}(3)$ version of the NJL model which includes scalar-pseudoscalar (chiral invariant) and the t'Hooft six fermion interactions [that models the axial $U(1)_{A}$ symmetry breaking] [36], with $\mathcal{L}_{\text {sym }}$ and $\mathcal{L}_{\text {det }}$ given by [37]

$$
\begin{gathered}
\mathcal{L}_{\text {sym }}=G \sum_{a=0}^{8}\left[\left(\bar{q} \lambda_{a} q\right)^{2}+\left(\bar{q} i \gamma_{5} \lambda_{a} q\right)^{2}\right], \\
\mathcal{L}_{\operatorname{det}}=-K\left\{\operatorname{det}\left[\bar{q}\left(1+\gamma_{5}\right) q\right]+\operatorname{det}\left[\bar{q}\left(1-\gamma_{5}\right) q\right]\right\},
\end{gathered}
$$

where $q=(u, d, s)^{T}$ represents a quark field with three flavors, $\hat{m}_{f}=\operatorname{diag}_{f}\left(m_{u}^{0}, m_{d}^{0}, m_{s}^{0}\right)$ is the corresponding (current) mass matrix, $\lambda_{0}=\sqrt{2 / 3} I$ where $I$ is the unit matrix in the three flavor space, and $0<\lambda_{a} \leq 8$ denote the Gell-Mann matrices. The coupling between the (electro) magnetic field $B$ and quarks, and between the effective gluon field and quarks, is implemented via the covariant derivative $D^{\mu}=\partial^{\mu}-i q_{f} A_{\mathrm{EM}}^{\mu}-i A^{\mu}$ where $q_{f}$ represents the quark electric charge $\left(q_{d}=q_{s}=-q_{u} / 2=-e / 3\right), A_{\mu}^{\mathrm{EM}}$ and $F_{\mu \nu}=\partial_{\mu} A_{\nu}^{\mathrm{EM}}-\partial_{\nu} A_{\mu}^{\mathrm{EM}}$ are used to account for the external magnetic field and $A^{\mu}(x)=g_{\text {strong }} \mathcal{A}_{a}^{\mu}(x) \frac{\lambda_{a}}{2}$ where $\mathcal{A}_{a}^{\mu}$ is the $\mathrm{SU}_{c}(3)$ gauge field. We consider a static and constant magnetic field in the $z$ direction, $A_{\mu}^{\mathrm{EM}}=\delta_{\mu 2} x_{1} B$. In the Polyakov gauge and at finite temperature the spatial components of the gluon field are neglected: $A^{\mu}=\delta_{0}^{\mu} A^{0}=-i \delta_{4}^{\mu} A^{4}$. The trace of the Polyakov line defined by $\Phi=\frac{1}{N_{c}}\left\langle\left\langle\mathcal{P} \exp i \int_{0}^{\beta} d \tau A_{4}(\vec{x}, \tau)\right\rangle\right\rangle_{\beta}$ is the Polyakov loop which is the exact order parameter of the $\mathbb{Z}_{3}$ symmetric/broken phase transition in pure gauge.

To describe the pure gauge sector an effective potential $\mathcal{U}(\Phi, \bar{\Phi} ; T)$ is chosen in order to reproduce the results obtained in lattice calculations [38],

$$
\begin{aligned}
\frac{\mathcal{U}(\Phi, \bar{\Phi} ; T)}{T^{4}}= & -\frac{a(T)}{2} \bar{\Phi} \Phi+b(T) \ln [1-6 \bar{\Phi} \Phi \\
& \left.+4\left(\bar{\Phi}^{3}+\Phi^{3}\right)-3(\bar{\Phi} \Phi)^{2}\right],
\end{aligned}
$$

where $a(T)=a_{0}+a_{1}\left(\frac{T_{0}}{T}\right)+a_{2}\left(\frac{T_{0}}{T}\right)^{2}, b(T)=b_{3}\left(\frac{T_{0}}{T}\right)^{3}$. The standard choice of the parameters for the effective potential $\mathcal{U}$ is $a_{0}=3.51, a_{1}=-2.47, a_{2}=15.2$, and $b_{3}=-1.75$.

The parameter $T_{0}$ is the critical temperature for the deconfinement phase transition within a pure gauge approach: it was fixed to a constant $T_{0}=270 \mathrm{MeV}$, according to lattice findings. Different criteria for fixing $T_{0}$ may be found in the literature, and one of them takes into account the quark backreaction effects on the Polyakov loop [39]. One should notice, however, that the behavior of the relevant physical quantities remains qualitatively the same.

The model being an effective one (up to the scale $\Lambda_{Q C D}$ ) and not renormalizable, we use as a regularization scheme a sharp cutoff, $\Lambda$, in 3-momentum space, only for the divergent ultraviolet integrals. The parameters of the model, $\Lambda$, the coupling constants $G$ and $K$ and the current quark masses $m_{u}^{0}$ and $m_{s}^{0}$ are determined by fitting $f_{\pi}, m_{\pi}$, $m_{K}$, and $m_{\eta^{\prime}}$ to their experimental values in a vacuum. 
Besides, the fifth quantity needed to adjust the parameters of the NJL sector of the model is an estimation of the quark condensate in the vacuum. We consider $\Lambda=602.3 \mathrm{MeV}$, $m_{u}^{0}=m_{d}^{0}=5.5 \mathrm{MeV}, \quad m_{s}^{0}=140.7 \mathrm{MeV}, G \Lambda^{2}=1.385$ and $K \Lambda^{5}=12.36$ as in [40].

The thermodynamical potential for the three flavor quark sector, $\Omega$, in the mean field approximation is written as

$$
\begin{aligned}
\Omega(T, B)= & 2 G \sum_{f=u, d, s}\left\langle\bar{q}_{f} q_{f}\right\rangle^{2}-4 K\left\langle\bar{q}_{u} q_{u}\right\rangle\left\langle\bar{q}_{d} q_{d}\right\rangle\left\langle\bar{q}_{s} q_{s}\right\rangle \\
& +\left(\Omega_{f}^{\mathrm{vac}}+\Omega_{f}^{\mathrm{mag}}+\Omega_{f}^{\mathrm{med}}\right),
\end{aligned}
$$

where the vacuum $\Omega_{f}^{\mathrm{vac}}$, the magnetic $\Omega_{f}^{\mathrm{mag}}$, the medium contributions $\Omega_{f}^{\text {med }}$ and the quark condensates $\left\langle\bar{q}_{f} q_{f}\right\rangle$ have been evaluated with great detail in $[41,42]$.

To obtain the mean field equations we must minimize the thermodynamical potential (3) with respect to the order parameters $\left\langle\bar{q}_{f} q_{f}\right\rangle, \Phi$ and $\bar{\Phi}$.

\section{THE CEP}

In the present section different scenarios obtained by choosing different values of the isospin and the strangeness chemical potentials are discussed. In terms of the baryon, charge and strangeness chemical potentials, $\mu_{B}, \mu_{Q}, \mu_{S}$ we have

$$
\begin{aligned}
\mu_{u} & =\frac{1}{3} \mu_{B}+\frac{2}{3} \mu_{Q}, \quad \mu_{d}=\frac{1}{3} \mu_{B}-\frac{1}{3} \mu_{Q}, \\
\text { and } \mu_{s} & =\frac{1}{3} \mu_{B}-\frac{1}{3} \mu_{Q}-\mu_{S} .
\end{aligned}
$$

No external magnetic field.-We first investigate the location of the CEP when no external magnetic field is present. We consider the models NJL and PNJL and the following different scenarios: (a) Equal quark chemical potentials as done in most calculations. This scenario corresponds to zero charge (or isospin) chemical potential and zero strangeness chemical potential $\left(\mu_{Q}=\mu_{S}=0\right)$. (b) Equal $u$ and $d$ quark chemical potentials and a zero strange quark chemical potential, corresponding to zero charge (isospin) chemical potential and a strangeness chemical potential equal to one-third of the total baryonic chemical potential $\left(\mu_{Q}=0 ; \mu_{S}=1 / 3 \mu_{B}\right)$. (c) Symmetric matter with equal amounts of quarks $u, d$, and $s$, known as strange matter $\left(\rho_{u}=\rho_{d}=\rho_{s}\right)$. (d) $\beta$-equilibrium matter corresponding to $\mu_{u}-\mu_{d}=\mu_{Q}=-\mu_{e}$ and $\mu_{d}=\mu_{s}$ $\left(\mu_{S}=0\right)$. The temperature, baryonic chemical potential and density of the CEPs are given in Table I.

We next analyze Fig. 1 to compare the different scenarios. The NJL results are shown just for reference. As already discussed in [43], the Polyakov loop shifts the CEP to higher temperatures and slightly smaller baryonic chemical potentials. Matter with the largest isospin asymmetry in this figure is represented by the $\beta$-equilibrium
TABLE I. Temperature, baryonic chemical potential and baryonic density at the CEP for NJL and PNJL. Different scenarios are considered.

\begin{tabular}{lcccccc}
\hline \hline & \multicolumn{3}{c}{$\mathrm{NJL}$} & \multicolumn{3}{c}{ PNJL } \\
\cline { 2 - 7 } & $\begin{array}{c}T \\
{[\mathrm{MeV}]}\end{array}$ & $\begin{array}{c}\mu_{B} \\
{[\mathrm{MeV}]}\end{array}$ & $\rho_{B} / \rho_{0}$ & $\begin{array}{c}T \\
{[\mathrm{MeV}]}\end{array}$ & $\begin{array}{c}\mu_{B} \\
{[\mathrm{MeV}]}\end{array}$ & $\rho_{B} / \rho_{0}$ \\
\hline$\mu_{u}=\mu_{d}=\mu_{s}$ & 68 & 948.5 & 1.71 & 155.4 & 873.8 & 1.87 \\
$\mu_{u}=\mu_{d} ;$ & 68 & 954.0 & 1.67 & 157.5 & 890.4 & 1.73 \\
$\quad \mu_{s}=0$ & & & & & & \\
$\rho_{u}=\rho_{d}=\rho_{s}$ & 73.8 & 1022.4 & 2.20 & 159.7 & 919.7 & 2.32 \\
$\beta$ equilibrium & 56.5 & 1005.6 & 1.48 & 140.1 & 915.1 & 1.73 \\
\hline \hline
\end{tabular}

results. The $\beta$-equilibrium CEP occurs for one of the largest chemical potentials, only slightly below the one obtained for strange matter. However, it is interesting to see that for $\beta$ equilibrium the CEP comes at lower temperatures. The reason becomes clear when analyzing the right panel of Fig. 1: the $\beta$-equilibrium matter being less symmetric is less bound and, therefore, the transition to a chirally symmetric phase occurs at a smaller temperature and density than the symmetric case.

In the following we analyze the effect of isospin asymmetry and we center our discussion on the PNJL model.

Isospin asymmetry.-In the previous section we have seen that the location of the CEP depends on isospin. In particular, it was shown that in $\beta$-equilibrium matter the CEP occurs at larger baryonic chemical potentials and smaller temperatures. To study in a more systematic way the effect of isospin on the CEP we take the $s$-quark chemical potential equal to zero and increase systematically $\mu_{d}$ with respect to $\mu_{u}$. We are interested in the $d$-quark rich matter as it occurs in HIC and neutron stars: isospin asymmetry presently attained in HIC corresponds to $\mu_{u}<\mu_{d}<1.1 \mu_{u}$, and neutron matter has $\mu_{d} \sim 1.2 \mu_{u}$. Larger isospin asymmetries are possible in $\pi^{-}$rich matter $[2,44]$.

In Fig. 2 the results for the CEP obtained for the set $\left(\mu_{d}\right.$, $\left.\mu_{u}, \mu_{s}=0\right)$ are shown. The red full point corresponds to the CEP with $\mu_{u}=\mu_{d}=\mu_{s}$. All other CEPs are calculated at $\mu_{s}=0$, and they all occur for $\rho_{s}=0$. The corresponding densities $\left(\rho_{u}, \rho_{d}, \rho_{s}\right)$ are given in Table II. Increasing the isospin asymmetry moves the CEP to smaller temperatures and larger baryonic chemical potentials (it can be understood with the same reasons as previously for the $\beta$ equilibrium case). Eventually, for an asymmetry large enough the CEP disappears. The threshold corresponds to $\mu_{d}=1.45 \mu_{u}$ and is represented in the graph by a star at $T=0$. This scenario corresponds to $\left|\mu_{u}-\mu_{d}\right|=\left|\mu_{I}\right|=$ $\left|\mu_{Q}\right|=130 \mathrm{MeV}$, below the pion mass, and, therefore, no pion condensation occurs under these conditions. The effect of pion condensation on the QCD phase diagram for finite chemical potentials has recently been discussed in $[2,28]$. We also remark that in [1], where the effect of 

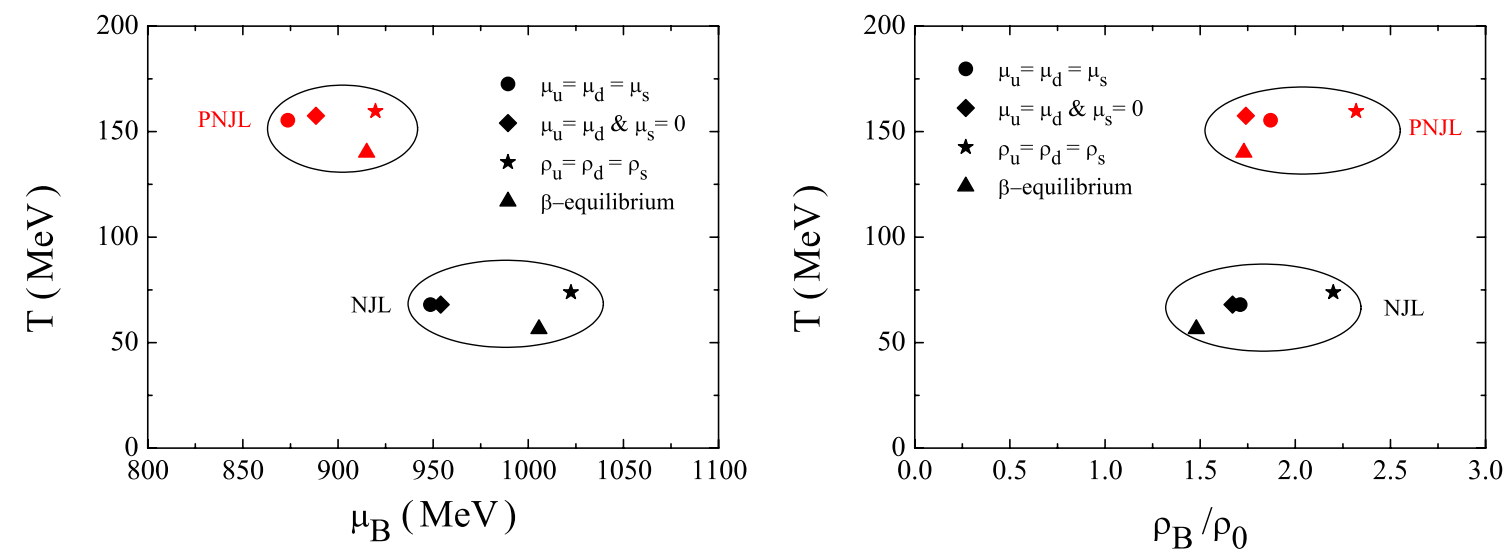

FIG. 1 (color online). Location of the CEP on a diagram $T$ versus the baryonic chemical potential (left) and $T$ versus the baryonic density (right) under different scenarios [all quark chemical potentials equal (circle), the strange quark chemical potential equal to zero (diamond), all quark densities equal (star), and $\beta$-equilibrium condition (triangle)] and models (NJL, PNJL). No external magnetic field is considered.
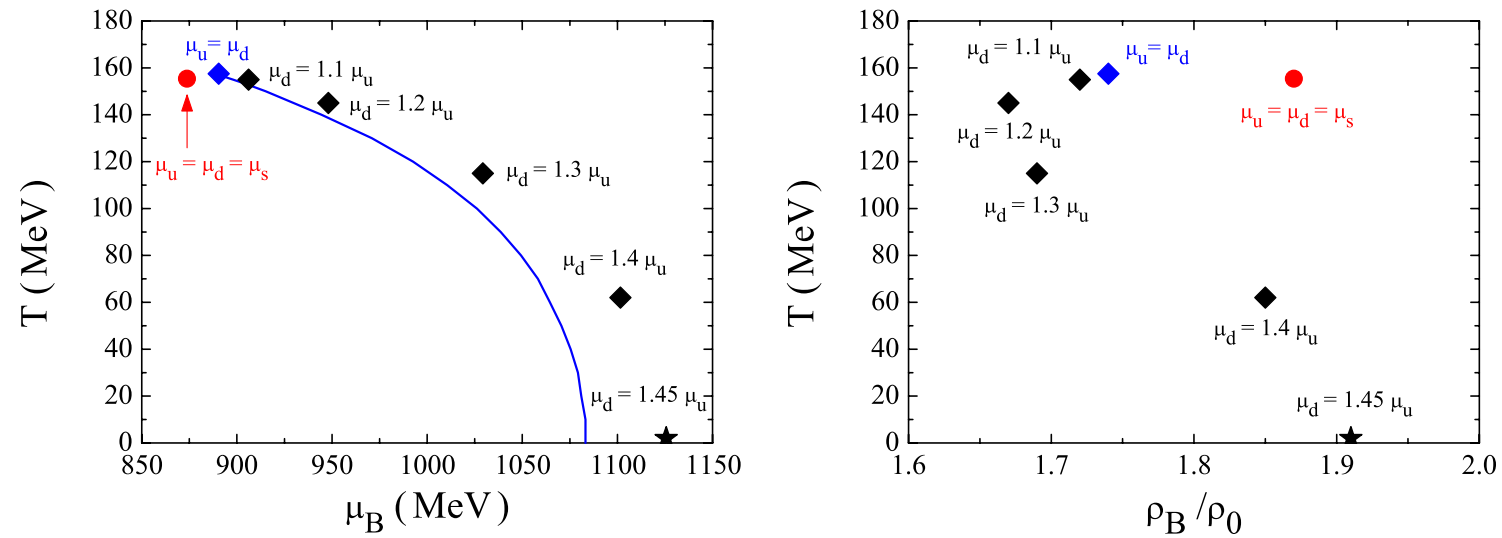

FIG. 2 (color online). Effect of isospin in the location of the CEP within the PNJL model. The full line is the first order phase transition line for zero isospin matter $\left(\mu_{d}=\mu_{u}, \mu_{s}=0\right)$. The strangeness chemical potential was always taken equal to zero. For $\mu_{d}>1.45 \mu_{u}$ no CEP exists. Isospin asymmetry presently attained in HIC corresponds to $\mu_{u}<\mu_{d}<1.1 \mu_{u}$ and neutron matter to $\mu_{d} \sim 1.2 \mu_{u}$.

isospin on the QCD phase diagram has also been discussed, a larger isospin chemical potential corresponds to a smaller baryonic chemical potential due to the definition of the baryonic chemical potential: in [1], the study was performed within the $\mathrm{SU}(2)$ quark-meson model, and the

TABLE II. The temperature, baryonic chemical potential, and $u(d)$ quark densities at the CEPs for different scenarios ratio $\mu_{d} / \mu_{d}$ with $\mu_{s}=0\left(\rho_{s}=0\right)$. The baryonic density is given in terms of the saturation density $\rho_{0}=0.16 \mathrm{fm}^{-3}$.

\begin{tabular}{lccccc}
\hline \hline CEP & $T[\mathrm{MeV}]$ & $\mu_{B}[\mathrm{MeV}]$ & $\rho_{B} / \rho_{0}$ & $\rho_{u} / \rho_{0}$ & $\rho_{d} / \rho_{0}$ \\
\hline$\mu_{d}=\mu_{u}$ & 157.5 & 890.4 & 1.74 & 1.50 & 1.50 \\
$\mu_{d}=1.1 \mu_{u}$ & 155 & 906 & 1.72 & 1.28 & 1.72 \\
$\mu_{d}=1.2 \mu_{u}$ & 145 & 948 & 1.67 & 1.05 & 1.95 \\
$\mu_{d}=1.3 \mu_{u}$ & 115 & 1029 & 1.69 & 0.75 & 2.25 \\
$\mu_{d}=1.4 \mu_{u}$ & 62 & 1102 & 1.85 & 0.50 & 2.50 \\
$\mu_{d}=1.45 \mu_{u}$ & $\sim 0$ & 1126 & 1.91 & 0.39 & 2.61 \\
\hline \hline
\end{tabular}

relation $\mu_{B}=3 \mu_{q}=\frac{3}{2}\left(\mu_{u}+\mu_{d}\right)$ was used; in the present work we get from Eq. (4) $\mu_{B}=\mu_{u}+2 \mu_{d}$. In both works the temperature of the CEP decreases when the isospin asymmetry increases.

In the left panel of Fig. 2, the CEP is shown for $T$ versus the baryonic density. For $\mu_{u}<\mu_{d}<1.2 \mu_{u}$ the baryonic density of the CEP decreases with asymmetry but for $\mu_{d} \gtrsim 1.2 \mu_{u}$ the opposite occurs and at the threshold $\left(\mu_{d}=1.45 \mu_{u}\right) \rho_{B} \sim 1.91 \rho_{0}$; see Table II.

External magnetic field.-In the following we study the effect of a static external magnetic field on the localization of the CEPs [45] previously calculated and plot the results in Fig. 3. The values for the CEPs are given in Table III. The red dots correspond to symmetric matter with $\mu_{u}=\mu_{d}=\mu_{s}$ and reproduce qualitatively the results previously obtained within the NJL in [6] to the PNJL model. The trend is very similar: as the intensity of the magnetic field increases, the transition temperature increases and the baryonic chemical 

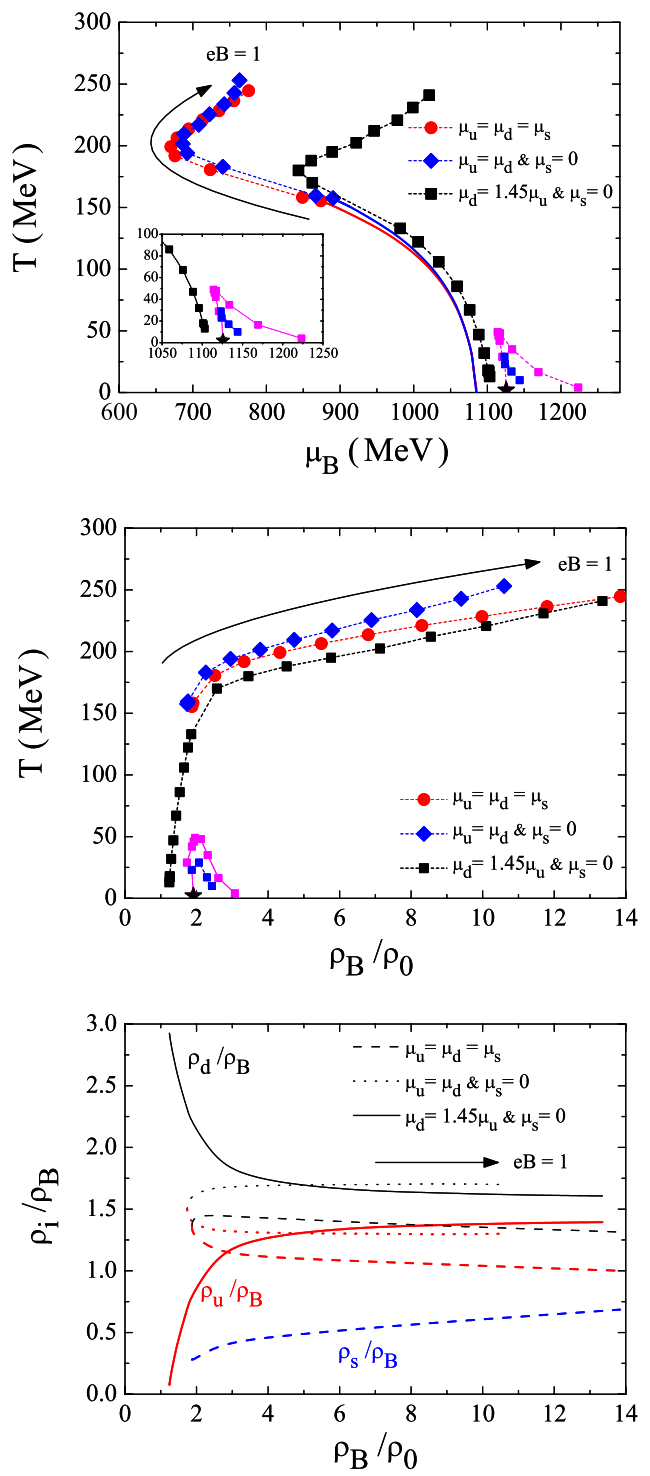

FIG. 3 (color online). Effect of an external magnetic field on the CEP's location within the PNJL model: $T^{\mathrm{CEP}}$ versus baryonic chemical potential (top panel) and baryonic density (middle panel). The full lines are the first order transitions at $e B=0$. Three scenarios are shown: $\mu_{u}=\mu_{d}=\mu_{s}$ (red dots), $\mu_{u}=\mu_{d}$; $\mu_{s}=0$ (blue diamonds) and $\mu_{d}=1.45 \mu_{u}, \mu_{s}=0$ (black squares) corresponding to the threshold isospin asymmetry above which no CEP occurs. In the last case for strong enough magnetic fields and low temperatures two or more CEPs exist at different temperatures for a given magnetic field intensity (pink and blue squares). The bottom panel shows the $u, d$ and $s$ quark fractions as a function of the baryonic density: dashed line corresponds to $\mu_{u}=\mu_{d}=\mu_{s}$, dotted line corresponds to $\mu_{u}=\mu_{d} ; \mu_{s}=0$ and full line corresponds to $\mu_{d}=1.45 \mu_{u} ; \mu_{s}=0$.

potential decreases until the critical value $e B \sim 0.4 \mathrm{GeV}^{2}$. For stronger magnetic fields both $T$ and $\mu_{B}$ increase. In the middle panel of Fig. 3 the CEP is given in a $T$ versus baryonic density plot. It is seen that when $e B$ increases from 0 to $1 \mathrm{GeV}^{2}$ the baryonic density at the CEP increases from $2 \rho_{0}$ to $14 \rho_{0}$.
TABLE III. The temperature, baryonic chemical potential and density at the CEPs for different values of the magnetic field and two different scenarios: $\mu_{u}=\mu_{d}=\mu_{s}$ and $\mu_{u}=\mu_{d} ; \mu_{s}=0$. The baryonic density is given in terms of the saturation density $\rho_{0}=0.16 \mathrm{fm}^{-3}$.

\begin{tabular}{lccccccc}
\hline \hline & \multicolumn{3}{c}{$\mu_{u}=\mu_{d}=\mu_{s}$} & & \multicolumn{3}{c}{$\mu_{u}=\mu_{d} ; \mu_{s}=0$} \\
\cline { 2 - 3 } \cline { 6 - 7 }$e B$ & $T$ & $\mu_{B}$ & & & $T$ & $\mu_{B}$ & \\
{$\left[\mathrm{GeV}^{2}\right]$} & {$[\mathrm{MeV}]$} & {$[\mathrm{MeV}]$} & $\rho_{B} / \rho_{0}$ & {$[\mathrm{MeV}]$} & {$[\mathrm{MeV}]$} & $\rho_{B} / \rho_{0}$ \\
\hline 0 & 155.4 & 873.8 & 1.87 & & 157.5 & 890.4 & 1.74 \\
0.1 & 158.2 & 848.9 & 1.90 & & 159.5 & 866.9 & 1.75 \\
0.2 & 180.6 & 723.8 & 2.51 & & 182.8 & 740.8 & 2.25 \\
0.3 & 191.8 & 675.7 & 3.33 & & 194.1 & 691.5 & 3.00 \\
0.4 & 199.2 & 670.2 & 4.33 & & 201.6 & 686.4 & 3.80 \\
0.5 & 206.4 & 678.6 & 5.49 & & 210.0 & 688.0 & 4.72 \\
0.6 & 213.6 & 694.5 & 6.80 & & 217.0 & 708.1 & 5.79 \\
0.7 & 221.0 & 713.3 & 8.30 & & 225.5 & 722.7 & 6.89 \\
0.8 & 228.4 & 735.5 & 9.98 & & 233.6 & 742.7 & 8.10 \\
0.9 & 236.4 & 755.6 & 11.80 & 242.8 & 756.5 & 9.40 \\
1 & 244.6 & 775.9 & 13.85 & 253.0 & 763.5 & 10.6 \\
\hline \hline
\end{tabular}

Taking the isospin symmetric matter scenario $\mu_{u}=\mu_{d}$ and $\mu_{s}=0$, the effect of the magnetic on the CEP is very similar to the previous one (see blue triangles in Fig. 3): $T$ is only slightly larger and the CEP baryonic density slightly smaller.

A very interesting case occurs for the very asymmetric matter scenario: a first order phase transition driven by the magnetic field takes place if $\mu_{d} \gtrsim 1.45 \mu_{u}$. Taking the threshold value $\mu_{d}=1.45 \mu_{u}$ it is seen that for $e B<$ $0.1 \mathrm{GeV}^{2}$ two CEPs may appear. In fact, for sufficiently small values of $e B$ the $T^{\mathrm{CEP}}$ is small and the Landau level effects are visible.

A magnetic field affects in a different way $u$ and $d$ quarks due to their different electric charges. A consequence is the possible appearance of two or more CEPs for a given magnetic field intensity. Two critical end points occur at different values of $T$ and $\mu_{B}$ for the same magnetic field intensity for fields $0.03 \lesssim e B \lesssim 0.07 \mathrm{GeV}^{2}$. Above $0.07 \mathrm{GeV}^{2}$ only one CEP remains. For stronger fields we get $T^{\text {CEP }}>100 \mathrm{MeV}$ : Landau level effects are completely washed out at these temperatures. In the lower panel of Fig. 3 we plot the $u$ and $d$ quark fractions corresponding to each CEP at different magnetic fields and for $\mu_{d}=1.45 \mu_{u}$ versus the baryonic density: it is seen that as the magnetic field becomes more intense the fraction of $u$ quarks comes closer to the $d$ quark fraction. This is due to the larger charge of the $u$ quarks and the fact that the quark density is proportional to the absolute value of the charge times the magnetic field intensity.

The right panel of Fig. 3 shows the $d$ (black), $u$ (red) and $s$ (blue) quark fractions as a function of the baryonic density at the CEPs for the three scenarios considered. When $\mu_{u}=\mu_{d}=\mu_{s}$ there is a strange quark fraction in the CEP which increases with the baryonic density. For the 
other two scenarios, the $u$ and $d$ quark fractions show a tendency to stabilize around $1.5 \rho_{B}$.

Finally, it is also important to point out that all three scenarios presented in Fig. 3 show an inverse magnetic catalysis at finite chemical potential and zero temperature once the critical temperature decreases with increasing $e B$ [46]. However, at large values of $e B$ the inverse magnetic catalysis tendency disappears and a magnetic catalysis takes place.

\section{CONCLUSIONS}

In the present study the location of the CEP on the QCD phase diagram was calculated within different scenarios in the framework of the SU(3) PNJL model. For reference some results obtained within the NJL model have also been shown.

Different scenarios have been considered, namely with respect to the isospin and strangeness content of matter. It was shown that for $\beta$-equilibrium matter the $\mathrm{CEP}$ occurs at smaller temperatures and densities. This scenario is of interest for neutron stars. However, the $T^{\mathrm{CEP}}$ calculated within PNJL seems too high to occur in a protoneutron star. These results, however, confirm previous calculations that indicate that a deconfinement phase transition in the laboratory will be more easily attained with asymmetric nuclear matter $[44,47]$. It was shown that for very asymmetric matter, in particular for $\mu_{d}>1.4 \mu_{u}$, no first order phase transition to a deconfined phase occurs. The disappearance of the CEP above a critical isospin chemical potential was also obtained in [2] where a GinzburgLandau approach was used to study the QCD phase structure.

We have next studied the effect of strong magnetic fields on the location of the CEP, generalizing the results of [6] to new nonsymmetric scenarios. For a zero $s$-quark and null isospin chemical potential, results very similar to the equal chemical potentials case were obtained. A more interesting situation was observed when analyzing very isospin asymmetric matter: in this case starting from a scenario having an isospin asymmetry above which the CEP does not exist for a zero external magnetic field it was shown that a sufficiently high external magnetic field could drive the system to a first order phase transition. The critical end point occurs at very small temperatures if $e B<0.1 \mathrm{GeV}^{2}$ and, in this case, a complicated structure with several CEP at different values of $\left(T, \mu_{B}\right)$ is possible for the same magnetic field, because the temperature is not high enough to wash out the Landau level effects. For $e B>0.1 \mathrm{GeV}^{2}$ only one CEP exists.

This is an important result because it shows that a strong magnetic field is able to drive a system with no CEP into a first order phase transition. In the present study we have explored the possibility that this occurs in a very isospin asymmetric system. A quite different situation could also give rise to a similar result: it has been shown that including a vector repulsive term in the quark model Lagrangian density it is possible to obtain a phase diagram with no first order chiral phase transition $[1,48]$. We may expect that a strong enough field would drive the system into a first order phase transition. This behavior would go along the results obtained in [49].

It has been shown that at zero baryonic chemical potential lattice QCD calculations predict a decreasing deconfinement critical temperature with an increase of $e B$ [16]. This behavior is not obtained within PNJL model [27]. Therefore, in order to confirm the present results it is important to include possible back reaction effects of the external magnetic field on the Polyakov loop.

\section{ACKNOWLEDGMENTS}

This work was partially supported by Projects No. PTDC/FIS/113292/2009 and No. CERN/FP/123620/ 2011 developed under the initiative QREN financed by the UE/FEDER through the program COMPETE_-"Programa Operacional Factores de Competitividade," by Grant No. SFRH/BD/51717/2011, by $\mathrm{CNPq} /$ Brazil and by FAPESC/Brazil.
[1] H. Ueda, T.Z. Nakano, A. Ohnishi, M. Ruggieri, and K. Sumiyoshi, Phys. Rev. D 88, 074006 (2013).

[2] H. Abuki, Phys. Rev. D 87, 094006 (2013).

[3] R. C. Duncan and C. Thompson, Astrophys. J. 392, L9 (1992); C. Kouveliotou et al., Nature (London) 393, 235 (1998).

[4] V. Skokov, A. Y. Illarionov, and V. Toneev, Int. J. Mod. Phys. A 24, 5925 (2009); V. Voronyuk, V. D. Toneev, W. Cassing, E. L. Bratkovskaya, V. P. Konchakovski, and S. A. Voloshin, Phys. Rev. C 83, 054911 (2011).

[5] T. Vachaspati, Phys. Lett. B 265, 258 (1991); K. Enqvist and P. Olesen, Phys. Lett. B 319, 178 (1993).
[6] S. S. Avancini, D. P. Menezes, M. B. Pinto, and C. Providência, Phys. Rev. D 85, 091901 (2012).

[7] K. G. Klimenko, Z. Phys. C 54, 323 (1992).

[8] D. Ebert and K. G. Klimenko, Nucl. Phys. A728, 203 (2003).

[9] E. J. Ferrer, V. de la Incera, and C. Manuel, Phys. Rev. Lett. 95, 152002 (2005).

[10] A. J. Mizher, M. N. Chernodub, and E. S. Fraga, Phys. Rev. D 82, 105016 (2010).

[11] B. Chatterjee, H. Mishra, and A. Mishra, Phys. Rev. D 84, 014016 (2011). 
[12] M. N. Chernodub, Phys. Rev. Lett. 106, 142003 (2011).

[13] M. Ferreira, P. Costa, and C. Providência, Phys. Rev. D 89, 036006 (2014).

[14] E. S. Fraga, Lect. Notes Phys. 871, 121 (2013).

[15] R. Gatto and M. Ruggieri, Lect. Notes Phys. 871, 87 (2013).

[16] G. S. Bali, F. Bruckmann, G. Endrödi, Z. Fodor, S. D. Katz, S. Krieg, A. Schäfer, and K. K. Szabó, J. High Energy Phys. 02 (2012) 044.

[17] M. D’Elia, Lect. Notes Phys. 871, 181 (2013).

[18] V. V. Braguta, P. V. Buividovich, T. Kalaydzhyan, S. V. Kuznetsov, and M. I. Polikarpov, Phys. At. Nucl. 75, 488 (2012).

[19] E.-M. Ilgenfritz, M. Kalinowski, M. Muller-Preussker, B. Petersson, and A. Schreiber, Phys. Rev. D 85, 114504 (2012).

[20] E.-M. Ilgenfritz, M. Muller-Preussker, B. Petersson, and A. Schreiber, arXiv:1310.7876.

[21] M. D'Elia, S. Mukherjee, and F. Sanfilippo, Phys. Rev. D 82, 051501 (2010).

[22] M. D'Elia and F. Negro, Phys. Rev. D 83, 114028 (2011).

[23] G. S. Bali, F. Bruckmann, G. Endrodi, Z. Fodor, S. D. Katz, and A. Schafer, Phys. Rev. D 86, 071502 (2012).

[24] J. Chao, P. Chu, and M. Huang, Phys. Rev. D 88, 054009 (2013).

[25] K. Fukushima and Y. Hidaka, Phys. Rev. Lett. 110, 031601 (2013).

[26] T. Kojo and N. Su, Phys. Lett. B 720, 192 (2013).

[27] M. Ferreira, P. Costa, D. P. Menezes, C. Providência, and N. Scoccola, Phys. Rev. D 89, 016002 (2014).

[28] T. Sasaki, Y. Sakai, H. Kouno, and M. Yahiro, Phys. Rev. D 82, 116004 (2010); J. O. Andersen and L. Kyllingstad, J. Phys. G 37, 015003 (2010).

[29] D. Ebert, K. G. Klimenko, M. A. Vdovichenko, and A. S. Vshivtsev, Phys. Rev. D 61, 025005 (1999).

[30] T. Inagaki, D. Kimura, and T. Murata, Prog. Theor. Phys. 111, 371 (2004).

[31] M. Ruggieri, L. Oliva, P. Castorina, R. Gatto, and V. Greco, arXiv:1402.0737.

[32] A. Ohnishi, H. Ueda, T.Z. Nakano, M. Ruggieri, and K. Sumiyoshi, Phys. Lett. B 704, 284 (2011).

[33] K. Fukushima, Phys. Lett. B 591, 277 (2004); C. Ratti, M. A. Thaler, and W. Weise, Phys. Rev. D 73, 014019 (2006).
[34] K. Fukushima, M. Ruggieri, and R. Gatto, Phys. Rev. D 81, 114031 (2010); R. Gatto and M. Ruggieri, ibid. 82, 054027 (2010); R. Gatto and M. Ruggieri, ibid. 83, 034016 (2011).

[35] W.-j. Fu, Phys. Rev. D 88, 014009 (2013).

[36] T. Hatsuda and T. Kunihiro, Phys. Rep. 247, 221 (1994); S. P. Klevansky, Rev. Mod. Phys. 64, 649 (1992).

[37] M. Buballa, Phys. Rep. 407, 205 (2005).

[38] S. Roessner, C. Ratti, and W. Weise, Phys. Rev. D 75, 034007 (2007).

[39] B.-J. Schaefer, J. M. Pawlowski, and J. Wambach, Phys. Rev. D 76 (2007) 074023; B.-J. Schaefer, M. Wagner, and J. Wambach, Phys. Rev. D 81, 074013 (2010); T. K. Herbst, J. M. Pawlowski, and B. J. Schaefer, Phys. Lett. B 696, 58 (2011).

[40] P. Rehberg, S. P. Klevansky, and J. Hüfner, Phys. Rev. C 53, 410 (1996).

[41] D. P. Menezes, M. B. Pinto, S. S. Avancini, A. P. Martínez, and C. Providência, Phys. Rev. C 79, 035807 (2009); D. P. Menezes, M. B. Pinto, S. S. Avancini, and C. Providência, Phys. Rev. C 80, 065805 (2009).

[42] S. S. Avancini, D. P. Menezes, and C. Providência, Phys. Rev. C 83, 065805 (2011).

[43] P. Costa, M. C. Ruivo, C. A. de Sousa, and H. Hansen, Symmetry 2, 1338 (2010); P. Costa, C. A. de Sousa, M. C. Ruivo, and H. Hansen, Europhys. Lett. 86, 31001 (2009); P. Costa, M. C. Ruivo, and C. A. de Sousa, Phys. Rev. D 77, 096001 (2008).

[44] R. Cavagnoli, C. Providência, and D. P. Menezes, Phys. Rev. C 83, 045201 (2011).

[45] P. G. Allen and N. N. Scoccola, Phys. Rev. D 88, 094005 (2013).

[46] J. O. Andersen and A. Tranberg, J. High Energy Phys. 08 (2012) 002.

[47] M. Di Toro, B. Liu, V. Greco, V. Baran, M. Colonna, and S. Plumari, Phys. Rev. C 83, 014911 (2011); G. Y. Shao, M. Di Toro, B. Liu, M. Colonna, V. Greco, Y. X. Liu, and S. Plumari, Phys. Rev. D 83, 094033 (2011); G. Y. Shao, M. Di Toro, V. Greco, M. Colonna, S. Plumari, B. Liu, and Y. X. Liu, Phys. Rev. D 84, 034028 (2011).

[48] N. Bratovic, T. Hatsuda, and W. Weise, Phys. Lett. B 719, 131 (2013).

[49] R. Z. Denke and M. B. Pinto, Phys. Rev. D 88, 056008 (2013). 